\title{
Computing an Arithmetic Constant Related to the Ring of Gaussian Integers
}

\author{
By F. Gramain and M. Weber
}

\begin{abstract}
We compute the analogue for $\mathbf{Z}[i]$ of Euler's constant, that is $\delta=\lim _{n \rightarrow+\infty} \delta_{n}$, where $\delta_{n}=\left(\Sigma_{2 \leqslant k \leqslant n} 1 / \pi r_{k}^{2}\right)-\log n$. For this purpose we give an estimate for$$
r_{k}=\min \{r \geqslant 0 \text {; there exists } z \in \mathbf{C} \text { such that } \operatorname{card}(\mathbf{Z}[i] \cap \bar{D}(z, r)) \geqslant k\},
$$

and we compute a great number of values of $r_{k}$.
\end{abstract}

0. Results and Notations. Let $\mathbf{Z}[i]=\{a+i b \in \mathbf{C} ; a, b \in \mathbf{Z}\}$ be the ring of Gaussian integers. For each integer $k \geqslant 2$ set

$$
r_{k}=\min \{r>0 ; \text { there exists } z \in \mathbf{C} \text { such that } \operatorname{card}(\mathbf{Z}[i] \cap \bar{D}(z, r)) \geqslant k\},
$$

where $\bar{D}(z, r)=\{w \in \mathbf{C} ;|w-z| \leqslant r\}$ is the closed disk with center $z$ and radius $r$.

Clearly, this minimum is attained and a closed disk with radius $r_{k}$ containing at least $k$ integer (i.e., Gaussian integer) points will be called a minimal disk $D_{k}$ and its boundary $\Gamma_{k}$ will be called a minimal circle. It is not known whether all the minimal disks with index $k$ are deduced from one another by an isometry of $\mathbf{R}^{2} \simeq \mathbf{C}$ which stabilizes the lattice $\mathbf{Z}[i]$. Nevertheless, Sections 1 and 2 deal with some properties of these disks. In particular, we prove in Section 2 an estimate of $r_{k}$ from which we deduce the existence of $\delta=\lim _{n \rightarrow+\infty} \delta_{n}$, where

$$
\delta_{n}=\left(\sum_{2 \leqslant k \leqslant n} \frac{1}{\pi r_{k}^{2}}\right)-\log n .
$$

This constant $\delta$ has been introduced by D. W. Masser (cf. [7] and [5]) in the study of entire functions which map $\mathbf{Z}[i]$ into $\mathbf{Z}[i]$. It is a bidimensional analogue of Euler's constant; it appears in a formula of the type of Stirling's formula and is related to the ring of Gaussian integers (see [7, Lemme 2] or [5, Proposition 3]) which leads to the conjecture that $\delta=1.822825 \ldots$ (cf. remark following Theorem 1 of [5]).

As no formula for $r_{k}$ is known, it has been necessary, in order to evaluate $\delta$, to compute a great number of values of $r_{k}$ with only the use of the above definition of $r_{k}$.

For this purpose, we have used a personal computer with a 6502 microprocessor ( 8 bits, $1 \mu s$ ) and 8 Kbytes memory. We have first developed various models in BASIC language which have enabled us to establish a table of the minimal disks $D_{k}$ for $k \leqslant 300$ (see Appendix 3), to compute fairly good approximations of $\delta_{1000}(1.80849)$, and then of $\delta_{1400}(1.81105$ : compare with the final result below!) in order to define

Received December 13, 1982.

1980 Mathematics Subject Classification. Primary 10E05, 10-04, 40-04. 
the optimal specifications of the final program. (All appendices are in the supplements section at the end of this issue.)

Indeed, as the BASIC interpreter used floating-point rounded values with little known errors, it was impossible to guarantee more than 4 decimal digits for the final result. Then, as all computed values were rational numbers (Propositions 1 and 2) with easily bounded sizes, we could develop a program in 6502 code working only with positive fixed-point values and multiprecision routines. With this method the accuracy can be as good as needed and the error computation is rigorous.

After approximately 175 hours of computation we obtained the following results

$$
1.811090114<\delta_{1400}<1.811090234
$$

and

$$
21.05521294<r_{1400}<21.05521402
$$

which, considering the estimates of Section 2 give

$$
1.811447299<\delta<1.897327117
$$

and the constant $\lambda_{0}$ of [7] therefore satisfies

$$
0.1707339<\lambda_{0}<0.1860446 \text {. }
$$

In particular, we have $\lambda_{0}>1 / 6$ (conjecture of [7]), but even if we notice that the lower bound for $\delta$ is better than its upper bound, we cannot yet assert that the above conjecture about the value of $\delta$ (which would lead to $\lambda_{0}=1 / 2 e=0.183939 \ldots$ ) is reasonable. Indeed, the estimates of Section 2 allow us to see that, to obtain $\delta$ with an accuracy of $10^{-n}$ (for $n \geqslant 4$ ), it is necessary to compute $\delta_{k}$ for $k$ near $50 \times 10^{3 n}$. So it seems impossible, even with big recent computers, to obtain the first 4 digits of $\delta$, unless the algorithm of calculation of $r_{k}$ or the estimate of $r_{k}$ are improved, or another way of computing $\delta$ is found.

1. Some Properties of Minimal Disks. In this section, two simple geometrical properties of minimal disks are given. With the notations of Section 0 we have

Proposition 1. Let $\Gamma_{k}$, with $k \geqslant 3$, be a minimal circle. If $\Gamma_{k}$ does not pass through (at least) 3 integer points, then $\Gamma_{k}$ has one diameter with integer extremities. This diameter is parallel to the real or imaginary axis and its length is an odd integer each of whose prime factors is congruent to 3 modulo 4.

Such a minimal circle will be called exceptional. One can verify that, for $3 \leqslant k \leqslant 1500$, there is no exceptional disk. For this purpose it is sufficient to compute the number $k$ of integer points belonging to the closed disk of center $1 / 2$ and of diameter $2 r$, where $2 r$ is an odd integer whose prime factors are all congruent to $3(\bmod 4)$ and satisfying $2 r<44$ (according to Proposition 3$)$. If one has $r$ $<\sqrt{(k-1) / \pi}$ one compares $r$ with $r_{k}^{\prime \prime}=\min _{l \geqslant k} r_{l}^{\prime}$, where

$$
\begin{array}{r}
r_{k}^{\prime}=\min \{r>0 ; \text { there exists } z \in \mathbf{C} \text { such that } \operatorname{card}(\bar{D}(z, r) \cap \mathbf{Z}[i])=k \\
\text { and } \operatorname{card}(\partial D(z, r) \cap \mathbf{Z}[i]) \geqslant 3\}
\end{array}
$$

is given by the BASIC program of Appendix 1 or the final program. This work is given in full detail in Appendix 2. 
However, there exist minimal disks $\Gamma_{k}$ with $k \geqslant 3$, whose one diameter is the segment parallel to the real axis connecting 2 integer points, and with an odd length (for instance $\Gamma_{22}$, with radius $\left.r_{22}=5 / 2\right)$. But in each known case $(k \leqslant 1500), \Gamma_{k}$ passes through at least 3 (therefore 6 by symmetry) integer points. Thus, it is natural to state the following:

CONJECTURE. For $k \geqslant 3$, any minimal circle $\Gamma_{k}$ passes through at least 3 integer points.

Proof of Proposition 1. That a minimal circle passes through at least 2 integer points is obvious.

Let $\Gamma$ be a circle passing through exactly 2 integer points $a$ and $b$ not on the same diameter. The radius $r$ of $\Gamma$ satisfies $2 r>|a-b|$ and if $\omega$ denotes the center of $\Gamma$ one has

$$
\max \{|\omega-\zeta| ; \zeta \in \mathbf{Z}[i] \cap \bar{D}(\omega, r), \zeta \neq a, \zeta \neq b\}=r-\varepsilon<r .
$$

Let $\omega^{\prime}$ be a point inside the triangle $\omega a b$ lying on the perpendicular from $\omega$ to $[a b]$, and such that $\left|\omega-\omega^{\prime}\right| \leqslant \varepsilon / 2$. The triangle inequality shows that the closed disk with center $\omega^{\prime}$ and radius $\left|\omega^{\prime}-a\right|<r$ contains the same integer points as $\bar{D}(\omega, r)$, so $\Gamma$ is not minimal.

Let us now study the case where $\Gamma$ has a diameter with integer extremities, for instance $a$ and $b$. (Note that the circle $\Gamma$ is not assumed to be minimal.) If $b-a \notin \mathbf{R} \cup i \mathbf{R}$, then the center $\omega=(a+b) / 2$ of $\Gamma$ belongs to $\frac{1}{2} \mathbf{Z}[i]$, thus, by symmetry with respect to the line $\omega+i \mathbf{R}$ or $\omega+\mathbf{R}$, the circle $\Gamma$ passes through at least 4 integer points. If $b-a \in 2 \mathbf{Z} \cup 2 i \mathbf{Z}$, then $\omega \in \mathbf{Z}[i]$ and it is easily seen (using a rotation of center $\omega$ and angle $\pi / 2$ ) that $\Gamma$ passes through at least 4 integer points.

In the only remaining case, we can suppose that $\Gamma$ has center $1 / 2$ and radius $m+1 / 2$ with $m$ an integer $\geqslant 1$. Then, the only integer points of $\Gamma$ are $-m$ and $m+1$ if and only if $(2 m+1)^{2}$ is not a sum of 2 squares, i.e., (see [6, Theorem 278]) if the prime factors of $2 m+1$ are all congruent to $3(\bmod 4)$. Q.E.D.

It is also possible to get more information on the position of the integer points of a minimal circle.

Proposition. 2. If a minimal circle passes through (at least) 3 integer points, then it is circumscribed to some triangle with integer vertices and angles $\leqslant \pi / 2$.

Proof. Let $D$ be a closed disk with boundary $\Gamma$ passing through at least 3 integer points and set $E=\Gamma \cap \mathbf{Z}[i]$. Suppose that each triplet of points in $E$ forms a triangle with one angle $>\pi / 2$. Then the convex hull of $E$ does not contain the center $\omega$ of $\Gamma$, for otherwise, by Carathéodory's theorem (cf. [1, Vol. 3, 11.1.8.6]), the center $\omega$ would be contained in the convex hull of 3 points of $E$, which would thus form a triangle with angles $\leqslant \pi / 2$.

Let $[a, b]$ be the chord of $\Gamma$ with extremities in $E$ and maximal length: Every point in $E$ is on the other side of $[a, b]$ with respect to $\omega$. Then, it is clear that any disk translated from $D$ by a vector orthogonal to $[a, b]$ directed from $\omega$ to $[a, b]$, and sufficiently small, will contain all the integer points of $D$ in its interior; thus $\Gamma$ is not minimal. Q.E.D. 
2. Estimate of $r_{k}$ and Existence of $\delta$. The values of $r_{k}$ which we computed show that the following upper bound is rather good.

Proposition 3. For each integer $k \geqslant 2$ one has $r_{k}<\sqrt{(k-1) / \pi}$.

Proof. By a classical result ([8, no. 452, p. 151] or [5, Proposition 1]), there exists a closed disk of radius $((k-1) / \pi)^{1 / 2}$ containing at least $k$ integer points. We thus have $r_{k}^{2} \leqslant(k-1) / \pi$. But it follows from Propositions 1 and 2 that $r_{k}^{2} \in \mathbf{Q}$ and, as $\pi$ is irrational, the inequality is strict. Q.E.D.

The following lower bound for $r_{k}$ is a refinement of classical calculations (see, for instance, [4]).

PROPOSITION 4. Let $k$ be the number of integer points lying in a closed disk of radius $r \geqslant 2$. Then

$$
\begin{aligned}
k< & \pi r^{2}+2\left(\sqrt{2}+\frac{4}{\sqrt{5}}-2\right) r+27-\frac{8 \sqrt{2}}{3 \sqrt{5}}-\frac{10 \sqrt{5}}{3}+\frac{1}{r}\left(\frac{2 \sqrt{2}+20 \sqrt{5}}{3}\right) \\
& -\frac{1}{r^{2}}\left(\left(\frac{6}{5}\left(\frac{7}{8}\right)^{5 / 2}-1\right)\left(\frac{2 \sqrt{2}}{\sqrt{5}}-1\right)+\frac{5}{4}\left(6\left(\frac{11}{16}\right)^{5 / 2}-5\right)(\sqrt{5}-2)\right) \\
& +\frac{1}{r^{3}}\left(\frac{1}{\sqrt{2}}\left(\frac{6}{5}\left(\frac{7}{8}\right)^{5 / 2}-1\right)+\frac{5 \sqrt{5}}{2}\left(6\left(\frac{11}{16}\right)^{5 / 2}-5\right)\right) .
\end{aligned}
$$

For $r \geqslant 21$, one can replace on the right-hand side $(7 / 8)^{5 / 2}$ by $(881 / 882)^{5 / 2}$ and $(11 / 16)^{5 / 2}$ by $(1759 / 1764)^{5 / 2}$.

Remark. This obviously gives a lower bound for $r_{k}$. If the above result is written in the form $k<\pi r^{2}+2 \alpha r+f(r)$, it is easily seen that $f$ is a decreasing function of $r$ and we can use this inequality in the following manner: For $k \geqslant k_{0}$ the radius $r_{k}$ is greater than the positive root of the trinomial $\pi X^{2}+2 \alpha X+f(\rho)-k$, where $\rho$ satisfies $0<\rho \leqslant r_{k_{0}}$. This lower bound is much less precise than the upper bound of Proposition 3 (as a matter of fact it is efficient for any disk, either minimal or not). Nevertheless, if $k$ is not too big $\left(k \leqslant 1.36410^{7}\right)$ this lower bound is better than the best known results taken from the work of $\mathrm{H}$. Chaix [2] on convex compact sets.

In order to obtain Proposition 4 the following calculation will be used:

LEMMA. The area $T_{1}$ of the convex curvilinear right-angled and isosceles triangle with sides 1 and hypotenuse of radius of curvature $r$ is

$$
T_{1}=\frac{1}{2}-\frac{r}{\sqrt{2}}\left(1-\frac{1}{2 r^{2}}\right)^{1 / 2}+\frac{r^{2}}{2} \operatorname{Arcsin} \frac{\sqrt{2}\left(1-1 /\left(2 r^{2}\right)\right)^{1 / 2}}{r} .
$$

The area $T_{2}$ of the convex curvilinear right-angled triangle with sides 1 and 2 and hypotenuse of radius of curvature $r$ is

$$
T_{2}=1-\frac{r \sqrt{5}}{2}\left(1-\frac{5}{4 r^{2}}\right)^{1 / 2}+\frac{r^{2}}{2} \operatorname{Arcsin} \frac{\sqrt{5}\left(1-5 /\left(4 r^{2}\right)\right)^{1 / 2}}{r}
$$

Proof. This is a straightforward computation of integrals.

Proof of Proposition 4. The closed disk $D$ with center $x+i y$ and radius $r$ is the disjoint union of $\{x+i y\}$ and of the 4 quarter disks

$Q_{1}=\{z=\xi+i \eta \in D ; \xi>x, \eta \geqslant y\}, \quad Q_{2}=i Q_{1}, \quad Q_{3}=-Q_{1}$ and $Q_{4}=-i Q_{1}$. 


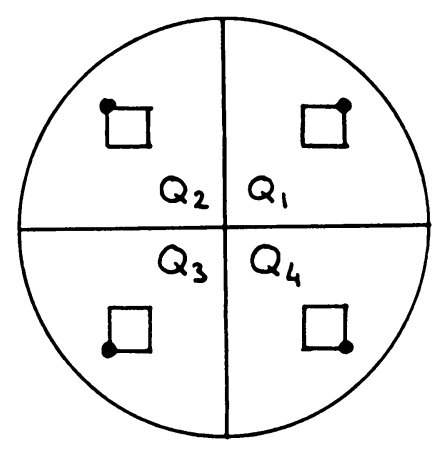

Figure 1

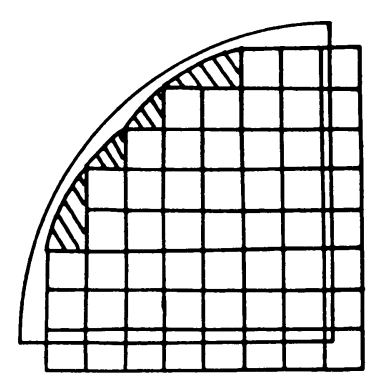

FIGURE 2

For each integer point belonging to $Q_{1}$ (resp. $Q_{2}$, resp. $Q_{3}$, resp. $Q_{4}$ ), construct the square with unit sides parallel to the real and imaginary axes with vertex equal to the above-mentioned integer point, and lying down left (resp. down right, resp. up right, resp. up left) as seen in Figure 1.

Let $A_{j}$ be the area of the union of the squares generated by the integer points of $Q_{j}$ and let $k$ be the number of integer points belonging to $D$. One has $k=\varepsilon+$ $\sum_{1 \leqslant j \leqslant 4} A_{j}$, where $\varepsilon=1$ if $x+i y \in \mathbf{Z}[i]$ and $\varepsilon=0$ otherwise.

The area $A_{j}$ is less than the sum of the area of $Q_{j}$ and the area of the union of the parts of the squares lying outside the radii bounding $Q_{j}$ minus the area of the union of curvilinear triangles whose vertices are the integer points nearest to the quarter circle bounding $Q_{j}$, whose rectilinear sides are some sides of squares generated by the integer points of $Q_{j}$, and whose hypotenuses are circular arcs of radius $r$. These triangles are indeed contained in $D$ since its radius is $r$, and they have been hatched in Figure 2 relating to $Q_{1}$.

If we study separately the case when $x+i y \in \mathbf{Z}[i]$ and its contrary, we get $k<\pi r^{2}+4 r+1-T$, where $T$ is the area of the union of the above triangles.

In order to bound $T$ from below, notice that the point of the first quadrant lying on the circle with center 0 and radius $r$ such that the tangent to the circle at this point has gradient -2 has abscissa $r \cos \left(\operatorname{Arctg} \frac{1}{2}\right)=2 r / \sqrt{5}$. Thus if the points $x+i y$ and $x+1+i y$ of the first quadrant belong to the closed disk $\bar{D}(0, r)$ and if $x \geqslant 2 r / \sqrt{5}$, then the point $x+i(y+2)$ also belongs to the disk. Similarly, if the points $x+i y$ and $x+1+i y$ of the first quadrant belong to the disk $\bar{D}(0, r)$ and if $x \geqslant r / \sqrt{2}$, then the point $x+i(y+1)$ also belongs to the disk. 


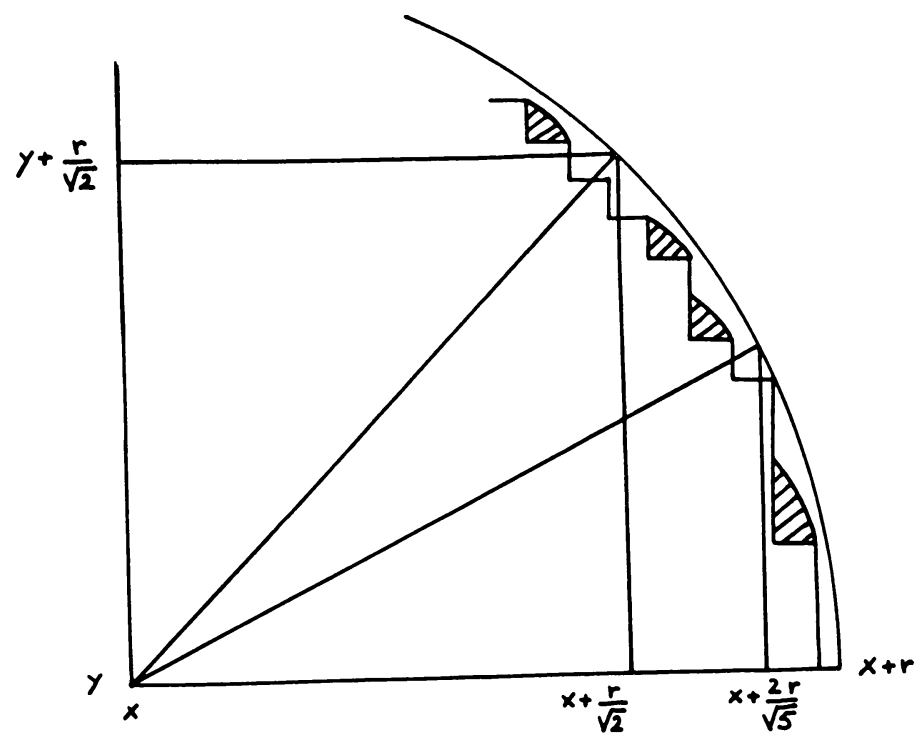

FIGURE 3

Consider again the disk of center $x+i y$ and radius $r$, and more precisely its first quarter $Q_{1}$ (for the other quarters make a similar calculation). Any segment with integer extremities and length 1 contained in the interval $[x+2 r / \sqrt{5}, x+r]$ generates a triangle with area $T_{2}$ (notation of the above lemma) contained in $D$. Similarly, any segment with integer extremities and length 1 contained in the interval $[x+r / \sqrt{2}, x+2 r / \sqrt{5}]$ generates a triangle with area $T_{1}$ contained in $D$. These triangles are hatched in Figure 3 and the area of their union is greater than $(r(1-2 / \sqrt{5})-2) T_{2}+(r(2 / \sqrt{5}-1 / \sqrt{2})-2) T_{1}$.

The same calculation can be applied to the second half-quarter, exchanging $x$ and $y$, but by Figure 3 it is easily seen that a few triangles in the neighborhood of the cuts $x+r / \sqrt{2}$ and $x+2 r / \sqrt{5}$ have been left aside. As one bounds $k$ from above by an increasing function of $r$, one can suppose without loss of generality that $D$ is minimal and thus that $r^{2}, x$ and $y$ are rational numbers.

If $x+2 r / \sqrt{5} \in \mathbf{Z}$, then $x+r / \sqrt{2} \notin \mathbf{Z}$, so that a triangle of area $T_{1}$ and a triangle of area $T_{2}$ can be added on either side of $x+2 r / \sqrt{5}$. Similarly, if $x+$ $r / \sqrt{2} \in \mathbf{Z}$, a triangle of area $T_{1}$ can be added in the neighborhood of $x+r / \sqrt{2}$ and another triangle of area $T_{1}$ near $x+2 r / \sqrt{5}$. If $x+2 r / \sqrt{5} \notin \mathbf{Z}$ and $x+r / \sqrt{2}$ $\notin \mathbf{Z}$, then a triangle of area $T_{1}$ can be added in the neighborhood of $x+2 r / \sqrt{5}$. Moreover, in the neighborhood of $x+r / \sqrt{2}$, a triangle with area at least $T_{1}$ can be added, but one must not add more than $T_{1} / 2$ because it can have a vertex of ordinate greater than $y+r / \sqrt{2}$, and therefore it may have been taken into account in the computation related to the second half-quarter. This allows us to add $8 \times \frac{3}{2} T_{1}$ to the lower bound for $T$, which becomes

$$
T \geqslant 8(r(1-2 / \sqrt{5})-2) T_{2}+8(r(2 / \sqrt{5}-1 / \sqrt{2})-1 / 2) T_{1} .
$$

Then finite expansions for $T_{1}$ and $T_{2}$ deduced from the lemma give the announced inequalities. Q.E.D. 
If $r$ is large enough, this upper bound for $k$ can be improved by dividing into 3, $4, \ldots$ parts each half- $Q_{j}$ eliciting triangles with areas $T_{3} \simeq 3 / 2, T_{4} \simeq 2, \ldots$, but, for $r$ near 20, the present cutting gives the best result.

Using the estimate for $r_{k}$ given by Propositions 3 and 4 we get the following

THEOREM. The sequence $\delta_{n}=\left(\sum_{2 \leqslant k \leqslant n} 1 / \pi r_{k}^{2}\right)-\log n$ is increasing and has a finite limit $\delta$ as $n$ tends to infinity.

Proof. From $r_{n+1}^{2}<n / \pi$ (Proposition 3) we deduce, for $n \geqslant 2$,

$$
\delta_{n+1}-\delta_{n}>\frac{1}{n}+\log n-\log (n+1)>0,
$$

so $\delta_{n}$ is an increasing sequence. Proposition 4 and Euler-Maclaurin's formula (see, for instance, [3, Chapter IX, Section 7]) give an upper bound for $\delta_{n}$. Further details are left to the reader. Q.E.D.

Actually, it is easy to obtain an estimate for $\delta-\delta_{n}$. Indeed, by Proposition 3 , one has

$$
\delta>\delta_{n}+\lim _{N \rightarrow+\infty}\left(\log \frac{n}{N+1}+\sum_{n \leqslant k \leqslant N} \frac{1}{k}\right)
$$

and Euler-Maclaurin's formula enables one to estimate this limit. Similarly, one gets an upper bound for $\delta$ using the lower bound for $r_{k}$ given by Proposition 4 for $1401 \leqslant k \leqslant 1.364 \times 10^{7}$, and then the lower bound $k<\pi r^{2}+30.84274723 \times r^{2 / 3}$ due to H. Chaix [2] for $k>1.364 \times 10^{7}$. By this process, we get the estimate of $\delta$ given in Section 0 from the computed value of $\delta_{1400}$ and we can see that $\delta-\delta_{n}=$ $O\left(n^{-2 / 3}\right)$.

\section{The Computation of $\delta_{N}$.}

(a) General Principles of the Programs. Our purpose is to compute $r_{n}$ for $n \leqslant N$ (see the definitions of $r_{n}^{\prime}$ and $r_{n}^{\prime \prime}$ following Proposition 1 in Section 1). We first compute $r_{n}^{\prime}$ and to this end we consider the circumcircles of triangles with integer vertices and radii $\leqslant \sqrt{(N-1) / \pi}$ (in (b) we will explain how to restrict the number of triangles to be considered): We compute the number of integer points contained in the associated disk.

For a given number of points $n<N$, the minimal radius of the studied disks containing exactly $n$ integer points is memorized, and for $n=N$ one memorizes the smallest radius giving at least $N$ integer points. When all the triangles have been studied, for a given $n$, there are 3 possibilities:

$(\alpha)$ Memory $r_{n}^{\prime}$ is empty (or strictly speaking contains 0 ).

( $\beta$ ) Memory $r_{n}^{\prime}$ contains a nonzero value. Then, either

$\left(\beta_{1}\right)$ memories $r_{n+t}^{\prime}(t \geqslant 1)$ contain 0 or values $\geqslant r_{n}^{\prime}$, or

$\left(\beta_{2}\right)$ there exists a memory $r_{n+t}^{\prime}(t \geqslant 1)$ containing a nonzero value $<r_{n}^{\prime}$.

Indeed, it is possible that none of the tested disks contains exactly $n$ integer points (case $(\alpha)$ arising for $n=3$, for example); it is also possible that each disk containing exactly $n$ integer points has a radius greater than the radius of some disks containing $n+t$ integer points (case $\left(\beta_{2}\right)$. For instance, the circumcircle of the triangle of vertices $-1,1$ and $3 i$ induces a disk containing $n=10$ integer points and with radius $5 / 3$, whereas the disk centered at $(1+i) / 2$ and with radius $\sqrt{5 / 2}<5 / 3$ contains 12 integer points). 
It is thus necessary to alter the table of $r_{n}^{\prime}$ in order to get the increasing sequence $r_{n}^{\prime \prime}$. For this purpose, we let $n$ run from $N-1$ to 2 , and we store in the memory of index $n$ the value $r_{n+1}^{\prime \prime}$ contained in the memory of index $n+1$ in case the memory of index $n$ contains 0 or a value greater than $r_{n+1}^{\prime \prime}$. Then it is sufficient to verify that there is no exceptional disk (cf. Section 1 and Appendix 2) and to compute $\delta_{N}$.

(b) Selection of the Circles to be Considered. Our purpose is to reduce the time of computation by avoiding, as far as possible, repeating the computations for triangles deduced from one another by the isometries of $\mathbf{R}^{2}$ which stabilize the lattice of Gaussian integers.

The circle considered here passes through the origin 0 and the 2 points $b$ and $c \in \mathbf{Z}[i]$ which can be picked up from different quadrants (the method used to construct the table in Appendix 3), or from the same quadrant. (This is the case of the model in Appendix 2 and of the final program which are described below).

It is easily verified that, as the triangle $0 b c$ has angles $\leqslant \pi / 2$, one can suppose $b$ and $c$ to be in the first quadrant. Moreover, $r_{N}<\sqrt{(N-1) / \pi}$; thus, one can assume that $|b| \leqslant|c| \leqslant L=2 \sqrt{N / \pi}$, and, by symmetry with respect to the first bisector of the axes, one can suppose that $\arg b \in[\pi / 4, \pi / 2]$.

The range of $b=x_{b}+i y_{b}$ is thus defined by

$$
\left\{\left(x_{b}, y_{b}\right) \in \mathbf{Z}^{2} ; 0 \leqslant x_{b} \leqslant L / \sqrt{2}, \max \left(1, x_{b}\right) \leqslant y_{b} \leqslant \sqrt{L^{2}-x_{b}^{2}}\right\} .
$$

For fixed $b$, one has $|c| \geqslant|b|$ and, since the angles of the triangle $0 b c$ are $<\pi / 2$, point $c$ is on the same side as 0 with respect to the perpendicular from $b$ to $[0, b]$ whose equation is

$$
x_{b} X+y_{b} Y=|b|^{2} \text {, }
$$

and which intersects the real axis at the point $|b|^{2} / x_{b}$.

So, for fixed $b$, the range of $c=x_{c}+i y_{c}$ is defined by

$$
\begin{array}{r}
\left\{\left(x_{c}, y_{c}\right) \in \mathbf{Z}^{2} ; 0 \leqslant x_{c} \leqslant \min \left(L,|b|^{2} / x_{b}\right), \min \left(\sqrt{L^{2}-x_{c}^{2}}, \frac{|b|^{2}-x_{b} x_{c}}{y_{b}}\right) \geqslant y_{c},\right. \\
y_{c} \geqslant\left[\begin{array}{ll}
0 & \text { if } x_{c}>|b| \\
\sqrt{|b|^{2}-x_{c}^{2}} & \text { otherwise }
\end{array}\right\},
\end{array}
$$

and $b \neq c$ (or equivalently $x_{b} y_{c}-x_{c} y_{b} \neq 0$ ).

It is easily seen that the circumcircle of the triangle $0 b c$ has center $x+i y$ and radius $r$ with $x=N(x) / D, y=N(y) / D$ and $r^{2}=N\left(r^{2}\right) / D^{2}$, where $N(x)=y_{c}|b|^{2}$ $-y_{b}|c|^{2}, N(y)=x_{b}|c|^{2}-x_{c}|b|^{2}, N\left(r^{2}\right)=|b|^{2}|c|^{2}|b-c|^{2}$ and $D=2\left(x_{b} y_{c}-x_{c} y_{b}\right)$.

One verifies without trouble that $r^{2}$ is a rational number with a denominator not greater than $D^{2} / 2$.

(c) Counting the Integer Points in a Disk.

( $\alpha$ ) Method used in the BASIC models. In the closed disk of center $x+i y$ and radius $r$ integer points belong to the straight lines parallei to the imaginary axis and with integer abscissa $k \in[x-r, x+r]$. Therefore they amount to

$$
\sum_{\substack{x \leq k \leq x+r \\ k \in \mathbf{Z}}}\left(\left[y+\sqrt{r^{2}-(x-k)^{2}}\right]-\left[y-\sqrt{r^{2}-(x-k)^{2}}\right]\right),
$$

where $[\cdot]$ denotes the integral part. 
With respect to BASIC rounded values, a parameter $\varepsilon$ (EP in Appendix 1) has been introduced in order not to leave out any integer point in this counting, by actually considering the disk of center $x+i y$ and radius $\sqrt{r^{2}+\varepsilon}$. The computation is valid if $\varepsilon$ is sufficiently small, and one chooses its values noticing that, from the denominators of $x, y$ and $r^{2}$, if the point $\alpha \in \mathbf{Z}[i]$ does not belong to the circle of center $x+i y$ and radius $r$, then one has ||$\alpha-(x+i y)|-r| \geqslant\left(2 L^{2}(L+1)\right)^{-1}$.

Nevertheless, the BASIC computation is not strictly justified (except for $N$ sufficiently small, in practice $N<500$ ). This is the reason why the final program and all needed arithmetical multiprecision routines have been developed directly in 6502 machine code.

( $\beta$ ) Method used in the final program. One computes the number of points in $D \mathbf{Z}[i]-D(x+i y)$ contained in the disk of center 0 and radius $D r$ (which is $\operatorname{card}(\mathbf{Z}[i] \cap \bar{D}(x+i y, r)))$.

Using an Euclidean division routine (64 bits dividend, 32 bits divisor) we compute the representative $X_{1}$ (resp. $\left.Y_{1}\right)$ of $N(x)$ (resp. $N(y)$ ) modulo $D$ belonging to [0,D , and the desired number of points is

$$
\sum_{X}\left(\left[\frac{Y_{1}+\sqrt{N\left(r^{2}\right)-X^{2}}}{D}\right]+\left[\frac{D-Y_{1}+\sqrt{N\left(r^{2}\right)-X^{2}}}{D}\right]\right),
$$

where the sum is taken over all $X$ in

$$
\begin{aligned}
\{X \in \mathbf{Z} ; X & \left.\equiv X_{1}(\bmod D), X_{1} \leqslant X \leqslant N\left(r^{2}\right)\right\} \\
& \cup\left\{X \in \mathbf{Z} ; X \equiv D-X_{1}(\bmod D), D-X_{1} \leqslant X \leqslant N\left(r^{2}\right)\right\} .
\end{aligned}
$$

The routine used for the square root gives the integral part of the exact square root, thus we obtain an accurate result.

(d) Values of $r_{k}^{-2}$ Memorized.

( $\alpha$ ) BASIC models. The computation of $r_{k}^{-2}=D^{2} / N\left(r^{2}\right)$ has been achieved with a 32 bit floating-point routine, but one does not know the sign of the error.

( $\beta$ ) Final program. It actually computes the integral part of $2^{32} r_{k}^{-2}=$ $2^{32} D^{2} / N\left(r^{2}\right)$ using an Euclidean division routine $\left(64\right.$ bit dividend, 32 bit $\mathrm{d}^{2}$ - sor and quotient). Thus, we get an approximation of $r_{k}^{-2}$ from below with an accuracy of $2^{-32}$.

(e) Summation of $r_{k}^{-2}$.

( $\alpha$ ) BASIC models. In view of the size of the memory the BASIC model of Appendix 1 has been used to compute $\sum_{2 \leqslant k \leqslant 1000} r_{k}^{-2}$. A second similar program was used to compute and to add up the $r_{k}^{-2}$ for $1001 \leqslant k \leqslant 1400$.

( $\beta$ ) Final program. For the same reasons mentioned above, the final program actually computes $\Delta_{N_{1}, N_{2}}=\sum_{N_{1} \leqslant k \leqslant N_{2}} 2^{32} r_{k}^{-2}$ (with $N_{1} \geqslant 6$ so that $2^{32} r_{k}^{-2}<2^{32}$ ). The work has been achieved in 3 steps giving

$$
\begin{gathered}
\Delta_{6,500} \simeq 69500353773, \quad \Delta_{501,1000} \simeq 9453770216 \text { and } \\
\Delta_{1001,1400} \simeq 4574912626 .
\end{gathered}
$$

As $\Delta_{2,5}=9.2^{32}$ one easily gets an approximation of $\delta_{1400}$ with an accuracy near 1395 $\pi^{-1} 2^{-32}$. 
Acknowledgment. The authors are grateful to Isabelle Plaud-Proietti for her help in translating the original manuscript into English.

Département de Mathématiques

Université $\mathbf{P}$. et $M$. Curie

4, Place Jussieu

F 75230 Paris Cedex 05, France

4, rue des Oseraies

F 91540 Mennecy, France

1. M. BeRger, Géométrie, CEDIC, Paris; Nathan Information, Paris, 1978.

2. H. ChalX, "Sur les points entiers voisins d'une courbe plane convexe-application," Journées de Théorie Analytique et Elémentaire des Nombres, Reims, 1981 and private communication.

3. J. DiEudonnE, Calcul Infinitésimal, Hermann, Paris, 1968.

4. A. O. GEL'FOND, "Sur les propriétés arithmétiques des fonctions entières," Tôhoku Math. J., v. 30, 1929, pp. 280-285.

5. F. Gramain, "Sur le théorème de Fukasawa-Gel'fond-Gruman-Masser," Séminaire Delange-PisotPoitou (Théorie des Nombres), 1980-1981, Birkhäuser, Basel, 1982.

6. G. H. HARDY \& E. M. WRIGHT, An Introduction to the Theory of Numbers, Clarendon Press, Oxford, 1971.

7. D. W. MASSER, “Sur les fonctions entières à valeurs entières," C. R. Acad. Sci. Paris Sér. A-B, v. 291, 1980, pp. 1-4.

8. G. Polya \& G. SzegO, Problems and Theorems in Analysis, Vol. II, Springer-Verlag, Berlin and New York, 1976. 


\title{
Supplement to \\ Computing an Arithmetic Constant Related to the Ring of Gaussian Integers
}

\author{
By F. Gramain and M. Weber
}

Appendix 1 : BASIC program for $\delta 1000$

$10 \mathrm{NP}=0 ; \mathrm{YC}=0: \mathrm{NX}=0: \mathrm{R2}=0: \mathrm{DN}=0: \mathrm{C}=0: \mathrm{F} 2=0: \mathrm{W} 2=0:$

$\mathrm{EP}=.00001: \mathrm{K}=0: \mathrm{X}=0: \mathrm{Y}=0: \mathrm{Wl}=0$

$20 \mathrm{~S}=\mathrm{O}: \mathrm{G}=\mathrm{O}: \mathrm{IR}=\mathrm{O}: \mathrm{L} 2=\mathrm{O}: \mathrm{L}=\mathrm{O}: \mathrm{U}=1: \mathrm{B} 2=\mathrm{O}: \mathrm{B}=\mathrm{O}: \mathrm{RM}=\mathrm{O}:$ $\mathrm{Ml}=\mathrm{O}: \mathrm{HI}=\mathrm{O}=\mathrm{M} 2=0: \mathrm{LX}=0: \mathrm{V} 2=0$

$30 \mathrm{XB}=\mathrm{O}: \mathrm{C} 2=\mathrm{O}: \mathrm{D}=2: \mathrm{YX}=\mathrm{O}: \mathrm{N}=1000: \mathrm{YB}=\mathrm{O}: \mathrm{BX}=\mathrm{O}: \mathrm{L} 1=\mathrm{O}:$ $\mathrm{Z}=0: \mathrm{P}=1.0001$

$40 \mathrm{DIM} R R(\mathrm{~N}): \operatorname{RR}(\mathrm{D})=4: \mathrm{L}=\mathrm{D} * \mathrm{SQR}(\mathrm{N} / \mathrm{\pi}): \mathrm{L} 2=\mathrm{I} * \mathrm{~L}: \mathrm{L} 1=\mathrm{L} 2 / 4$

100 FOR XB $=2$ TO L/SOR(D) : B2 $=\mathrm{XBEB}: \mathrm{LX}=\mathrm{XB}: \mathrm{IF} \mathrm{XB}=\mathrm{Z}$ THEN LX $=\mathrm{U}$

110 FOR YB $=$ LX TO SQR(L2-B2) : B = B2+YB*YB : PRDNT TID ; XB, YB

120 FOR XC $=2$ TO L $: \mathrm{BX}=\mathrm{BXEXC}: \mathrm{C2}=\mathrm{XCNXC}: \mathrm{YX}=\mathrm{YBNC}: \mathrm{Ml}=\mathrm{Z}:$

M2 $=(B-C 2) / \mathrm{P}:$ IF $\mathrm{M2}>\mathrm{Z}$ THEN M1 $=\operatorname{SQR}($ M2)

$130 \mathrm{PM}=\mathrm{Z}: \mathrm{HI}=\mathrm{P}(\mathrm{B}-\mathrm{XE*XC}) / \mathrm{YB}: \mathrm{FF}$ HI $<\mathrm{Z}$ THEN NEXT YB : NEXT : ФO TO 260

$140 \mathrm{PM}=\mathrm{SQR}(\mathrm{L} 2-\mathrm{C} 2): \mathrm{IF} \mathrm{RM}>\mathrm{HI}$ THEN RM $=\mathrm{HI}$

150 FOR YC $=\mathrm{INT}(\mathrm{RM})$ TO MI STEP $-\mathrm{U}: \mathrm{C}=\mathrm{C} 2+\mathrm{YC} * \mathrm{YC}$

$160 \mathrm{DN}=\mathrm{D}(\mathrm{YX}-\mathrm{XENYC}):$ IF DN $=Z$ THEN NEXT $:$ NEXT $:$ NEXT $:$ NEXT $:$ TO TO 260

$170 \mathrm{NX}=\mathrm{YHC}-\mathrm{YC} B \mathrm{~B}: \mathrm{NY}=\mathrm{BX}-\mathrm{XECC}: \mathrm{S}=\mathrm{NX}=\mathrm{NX}+\mathrm{NY} \pm \mathrm{NY}: \mathrm{G}=\mathrm{DN}: \mathrm{N} 2=\mathrm{N} / \mathrm{G}$

180 IF R2>L1 THEN NEXT : NEXT : NEXT : NEXT : GO TO 260

$190 \mathrm{Y}=\mathrm{NY} / \mathrm{DN}: \mathrm{X}=\mathrm{NX} / \mathrm{DN}: \mathrm{NP}=\mathrm{Z}: \mathrm{Fl}=\mathrm{SQR}(\mathrm{R} 2+\mathrm{EPP}): \mathrm{Wl}=\mathrm{W}-\mathrm{F} 1$

200 IF W1< $>\mathbb{N N T}$ (W1) THEN W1 $=\operatorname{TNT}($ Wl + U)

210 FOR $\mathrm{K}=\mathrm{W} 1 \mathrm{TO} \mathrm{X}+\mathrm{F} 1: \mathrm{F} 2=\mathrm{X}-\mathrm{K}: \mathrm{F} 2=\mathrm{SQR}(\mathrm{R} 2+\mathrm{FP}-\mathrm{F} 2 * \mathrm{~F} 2): \mathrm{W} 2=\mathrm{Y}-\mathrm{F} 2$

$220 \mathrm{NP}=\mathrm{NP}+\mathrm{INT}(\mathrm{Y}+\mathrm{F} 2)-\mathrm{INT}(\mathrm{W} 2): \mathrm{NEXT}$

230 IF $\mathrm{NP}>\mathrm{N}$ THEN $\mathrm{NP}=\mathrm{N}$

240 IR $=$ G/S : IF RR(NP) > IR THEN NEXT : NEXT : NEXT : NEXT : CO TO 260

$250 \mathrm{RR}(\mathrm{NP})=$ IR : NEXT : NEXT : NEXT : NEXT

260 OPEN $1:$ FOR K $=$ N TO U STEP $-\mathrm{U}:$ PRINT $=1$, RR $(\mathrm{K}):$ NEXT $:$ CLOSE $1:$ END

This first program computes the $r_{k}^{-2}$ and stores their values in the table $\mathrm{RR}(\mathrm{K})$, then in the file \# 1 . The latter is exploited by the program below which computes the $r_{k}^{n^{-2}}$ (the conjecture in $\S .1$ is that $r_{k}^{n}=r_{k}$ for $k \geqslant 3$ ) and then computes $\delta_{\mathrm{n}}$ for $\mathrm{n}=\mathrm{SM}$.

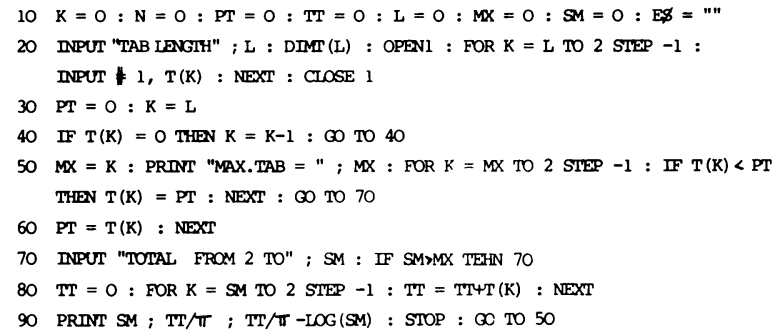




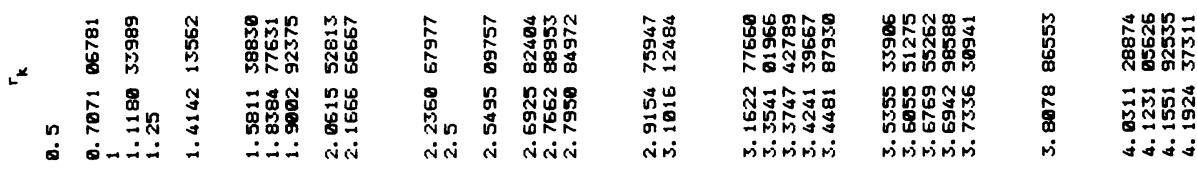

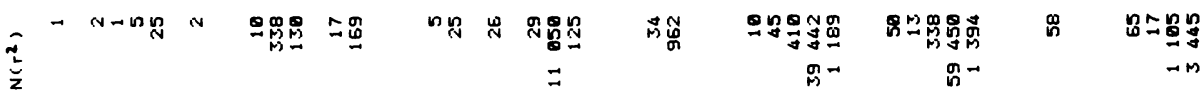

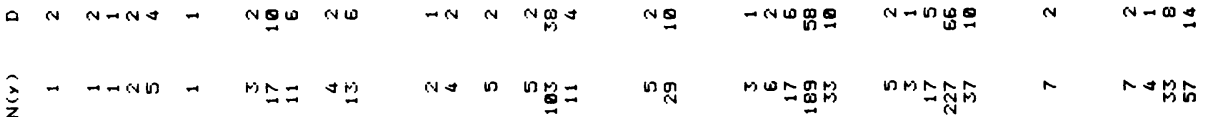

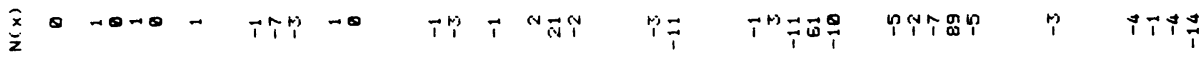

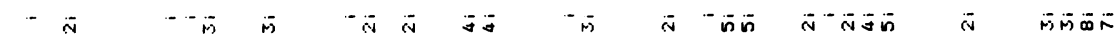

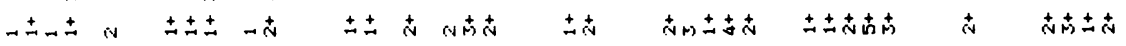

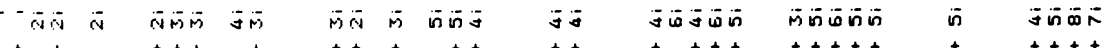

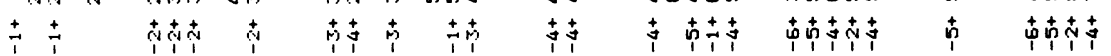
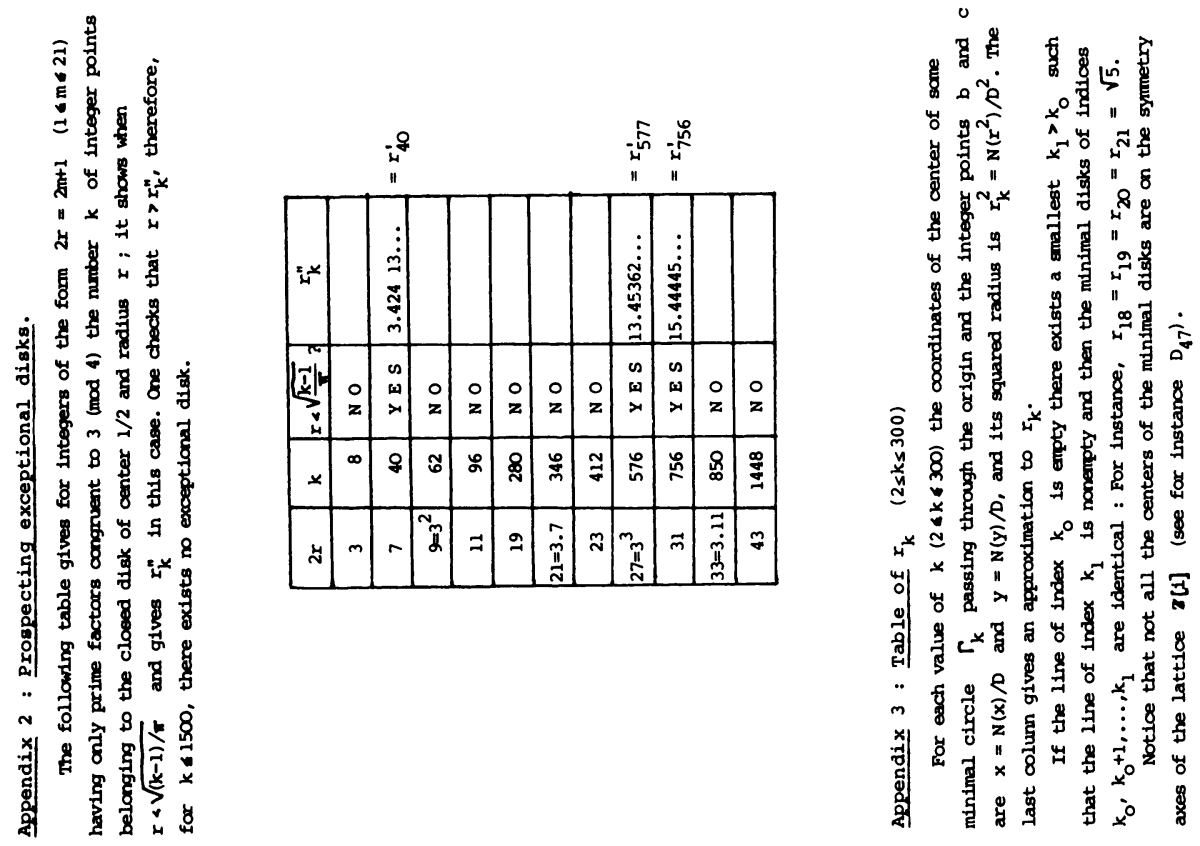


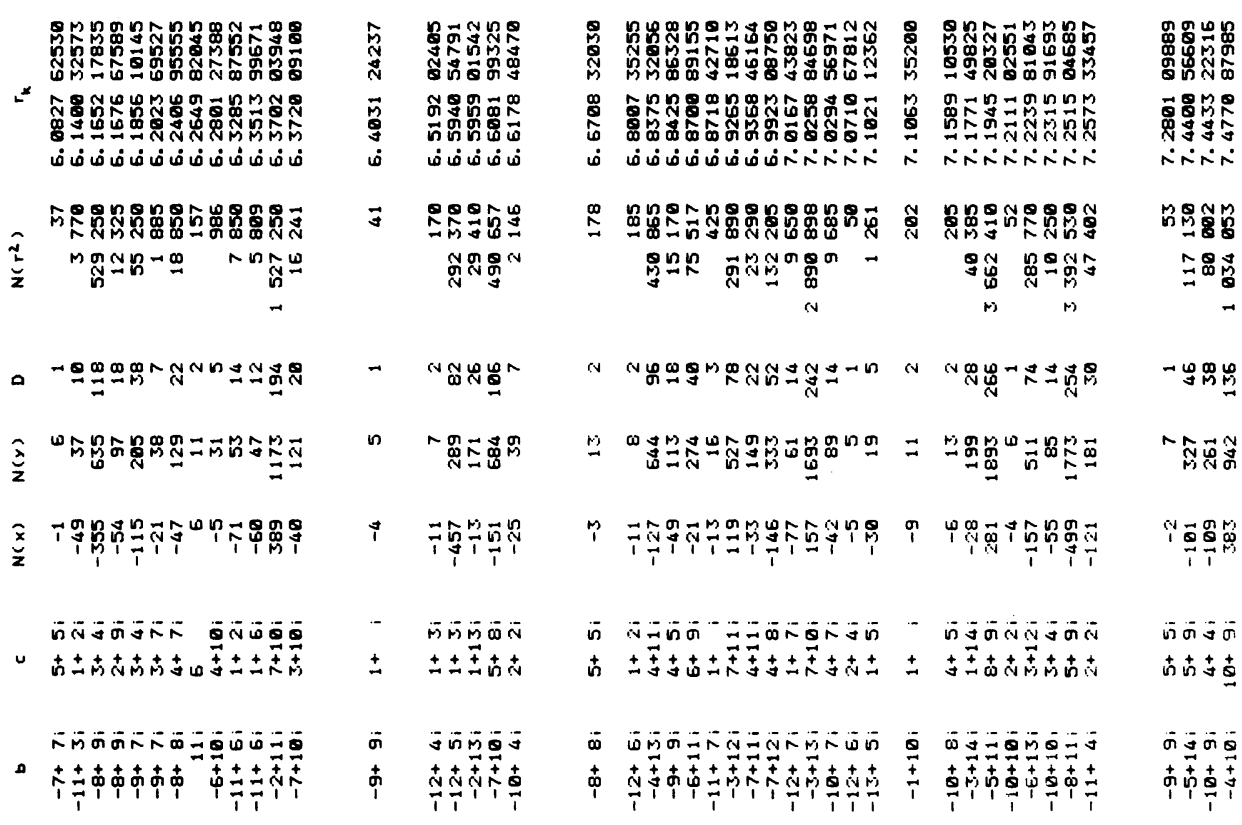

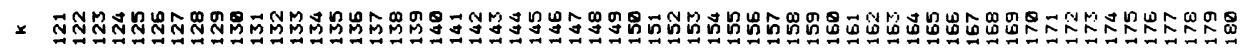
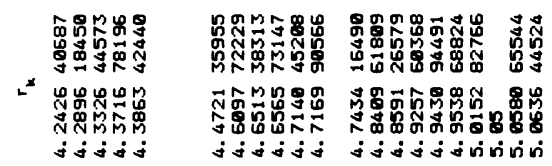

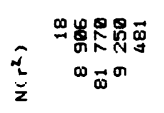

$\operatorname{Dannn}$

¿ "

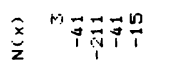

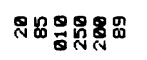

ถู

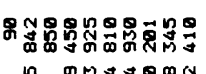

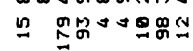

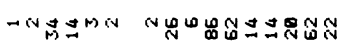

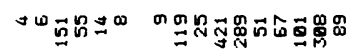

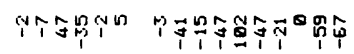

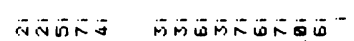

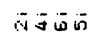

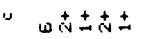

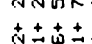

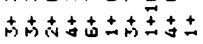

的影的

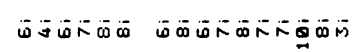

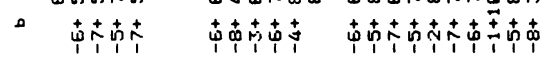
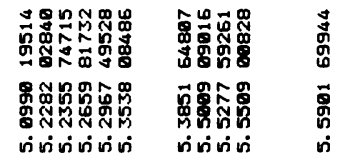

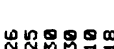

品角

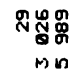

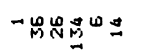

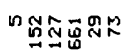

$\operatorname{lng}$

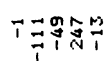

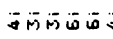

志古古古さ

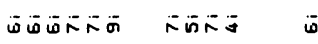

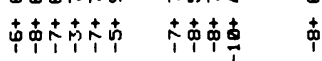

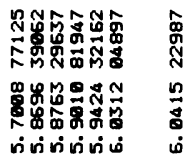

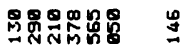

พN

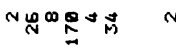

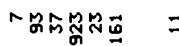

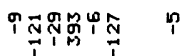

$\bar{N} \bar{N} \cdots \bar{n}$ in

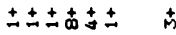

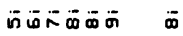

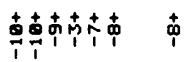

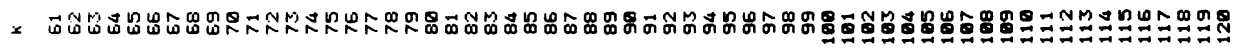




\begin{tabular}{|c|c|c|c|c|c|c|}
\hline 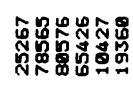 & 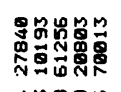 & 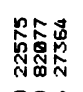 & 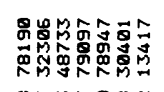 & $\begin{array}{c}n \\
\substack{n \\
\text { on }\\
}\end{array}$ & 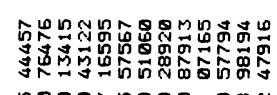 & 总 \\
\hline 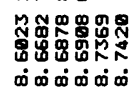 & 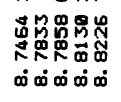 & 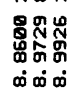 & 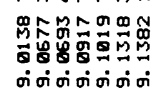 & 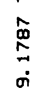 & 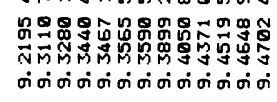 & 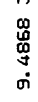 \\
\hline 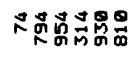 & 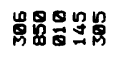 & 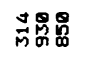 & 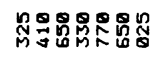 & $\hat{m}$ & 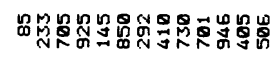 & \% \\
\hline 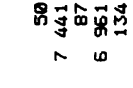 & 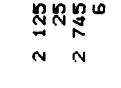 & $\begin{array}{l}\text { 昌n } \\
0 \\
9\end{array}$ & 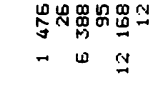 & & 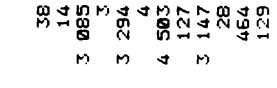 & \\
\hline 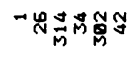 & 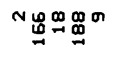 & NN & 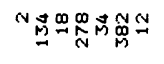 & N & 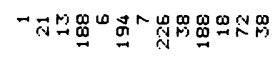 & - \\
\hline 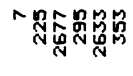 & 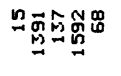 & 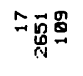 & 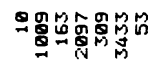 & $\stackrel{\mathscr{L}}{2}$ & 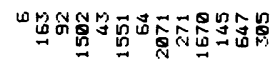 & $\sigma$ \\
\hline 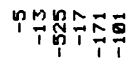 & 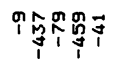 & 跨色 & 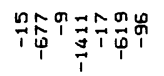 & $n$ & 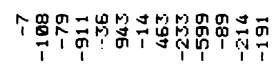 & $m$ \\
\hline $\begin{array}{lll} & \\
0\end{array}$ & 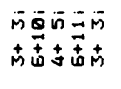 & 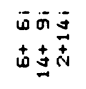 & 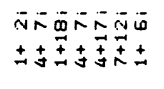 & $\sigma$ & 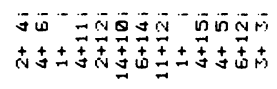 & $\begin{array}{l}\text { iे } \\
+ \\
\end{array}$ \\
\hline 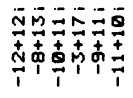 & 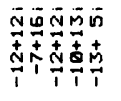 & 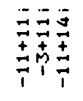 & 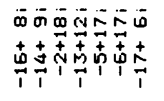 & $\underline{\underline{\theta}}$ & 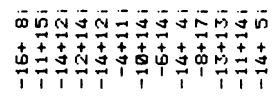 & 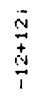 \\
\hline
\end{tabular}

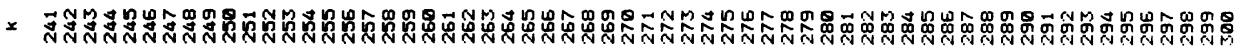

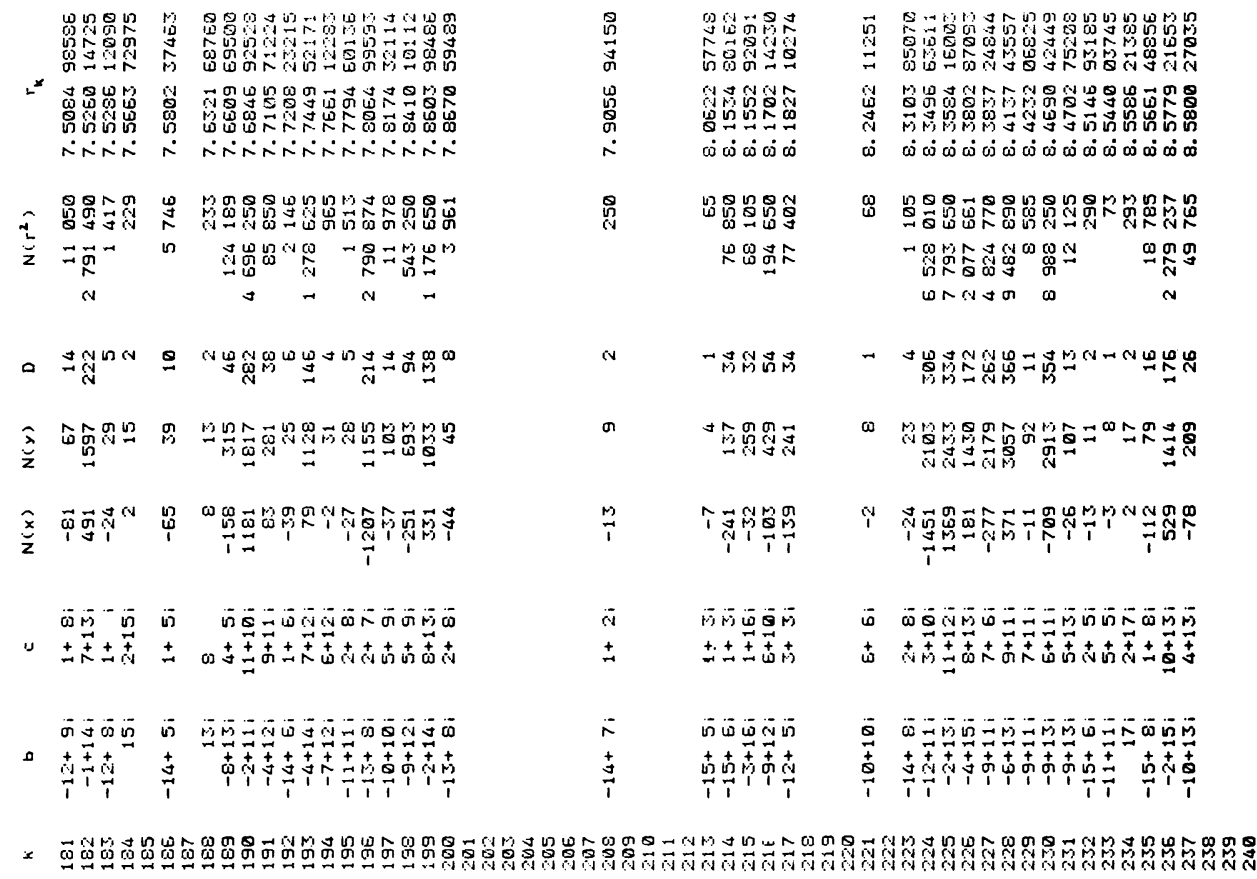

